\title{
WebWatch
}

\section{Fluish Dynamics}

As pandemics go, H1N1 fits into the category of a fairly manageable one (there is a vaccine, after all). Yet, the public perception of the illness is disturbingly inaccurate, thanks to its scary portrayal in the media. Paddling upstream against the current of misinformation about this important public health matter is the Influenza Research Database, which deals with research data and serves scientists in the field with timely information and computing utilities. The primary focus is on search/analysis tools, including a considerable amount of sequence information and protein structure data, a workbench for collecting online data (login required), and numerous standard sequence analysis tools. Links to influenza news sources round out the offerings.

[www.fludb.org]

\section{Notes of Prokaryotes}

Microscopes, antibiotics, vaccines, and biotechnology bring awareness of the microbial cosmos-in which the Earth is bathed - to those of us who perceive things macroscopically. For better or worse, microbes rule this bio-oasis we call home, so it's not surprising when they stage a protest from time to time. Unfortunately, if the "natives are restless," the results are sometimes catastrophic. Fortunately, Microbes.info's crack news team is on the job 24/7 to cover these uprisings as they occur. From bacteria involved in food and medicine to industrial production, Microbes.info keeps visitors abreast of goings-on in the bacterial kingdom. If you've got a need to know about the tiny world below, Microbes.info is the place to go.

[www.microbes.info]

\section{Dinging Dengue}

Divide and conquer. Anyone who has contributed to a distributed computing effort knows the principle of tackling an enormous computational problem by breaking it down into tiny, solvable pieces that are fed to thousands-or even millions - of personal computers for analysis. The protein folding problem (Folding@Home) and the search for extraterrestrial intelligence (SETI@Home) are

\section{The Winning Anther}

Speaking of microscopic domains, Nikon sponsors an annual photography contest for images taken through the 'scope that are as stunning as they are other-worldly. The winner for 2009's Small World Competition has just been announced (an anther from Arabidopsis thaliana, pictured here) and it-along with the many outstanding runnersup - are available for view at Nikon's easily navigable web site. Viewing the stunning images, one can debate whether art imitates life or vice versa,

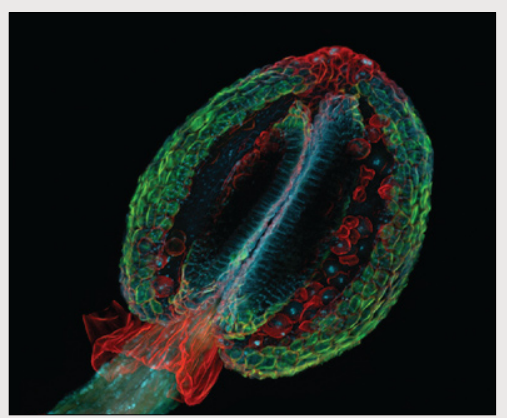

Heiti Paves @ 2009, Nikon Small World. but it probably doesn't matter. Life is beautiful in all its forms and Nikon Small World provides many reminders.

[www.nikonsmallworld.com]

two of the best-known projects. A new effort has surfaced that works on a more tractable problem than previous attempts, and it is likely to have positive effects on human health. Discovering Dengue Drugs Together assembles the computers of volunteers to help design drugs to neutralize an essential protease of the virus responsible for a deadly hemorrhagic fever. $40 \%$ of the world's population is at risk of the disease. At the University of Texas Medical Branch (UTMB) site, visitors can learn more about the project's progress and sign up to receive a program to install on their computers to contribute to the effort.

[www.utmb.edu/discoveringdenguedrugs-together]

\section{Floral Sense}

Combine the work of numerous volunteers and one part-time paid engineer and you'll probably be hard-pressed to duplicate the functionality of Calflora, the online go-to source of information about California plants. Considering the broad range of environments in the most populous U.S. state, the matter of comprehensive coverage is not a trivial one. There is a lot of territory here! Calflora meets the challenge of managing its information with sophisticated search functions that give users the ability to locate desired data in several ways. A standard search box linked to user- definable parameters sits on the opening page and helps users track down the plants they need. For those whose approach is more visual in nature, the numerous images are great and the map-based retrieval system provides an interface that anyone can understand. Despite the daunting pile of data it has to manage, Calflora succeeds. [http://calflora.org]

\section{The Eternal Question}

A site whose teaser URL derives less from the philosophical question that humans have asked about their existence for ages, than from Erwin Schrodinger's 1944 tome that formed the framework for investigating molecules as the basis of biology, What is Life takes a conceptual view of living things. It affords visitors the ability to explore topics ranging from our evolving concept of a gene to man, machine, and artificial life. If you're looking for links between biology and art, or you don't understand evolution and wonder what it's all about, What is Life may be the site for you.

[www.whatislife.com]

Written by Kevin Ahern, Ph.D.Please send web

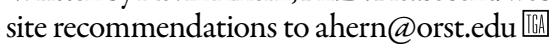

BioTechniques 47:1003 (December 2009)

doi 10.2144/000113313 